\title{
Creencias sobre la violencia y sus efectos en la prevalencia de la violencia en el noviazgo
}

\author{
Arturo Enrique Orozco Vargas; María del Rocío Mercado Monjardín; Georgina Isabel García López; \\ Arturo Venebra Muñoz; Ulises Aguilera Reyes
}

Cómo citar este artículo:

Orozco Vargas, A. E., Mercado Monjardín, M, R., García López, G. I., Venebra Muñoz, A., \& Aguilera Reyes, U. (2021). Creencias sobre la violencia y sus efectos en la prevalencia de la violencia en el noviazgo. Acta Colombiana de Psicología, 24(1), 154-166. https://www.doi.org/10.14718/ACP.2021.24.1.14

Recibido, junio 20/2019; Concepto de evaluación, septiembre 30/2020; Aceptado octubre 13/2020

\author{
Arturo Enrique Orozco Vargas \\ ORCID: https://orcid.org/0000-0002-2241-4234 \\ Universidad Autónoma del Estado de México, Toluca, México. \\ María del Rocío Mercado Monjardín \\ ORCID: https://orcid.org/0000-0002-9392-4100 \\ Universidad Autónoma del Estado de México, Toluca, México. \\ Georgina Isabel García López \\ ORCID: https://orcid.org/0000-0002-3370-0981 \\ Universidad Autónoma del Estado de México, Toluca, México. \\ Arturo Venebra Muñoz \\ ORCID: https://orcid.org/0000-0002-5406-1146 \\ Universidad Autónoma del Estado de México, Toluca, México. \\ Ulises Aguilera Reyes \\ ORCID: https://orcid.org/0000-0002-7563-9445 \\ Universidad Autónoma del Estado de México, Toluca, México.
}

\section{Resumen}

\begin{abstract}
La violencia en el noviazgo es un fenómeno multifactorial que requiere de estudios que profundicen respecto a los efectos que tienen las creencias culturales en el comportamiento tanto del agresor como de la víctima. Teniendo esto en cuenta, el objetivo de la presente investigación fue determinar la relación entre las creencias acerca de la violencia y la prevalencia de la violencia en el noviazgo. Para ello, se contó con una muestra de 420 estudiantes de dos universidades públicas mexicanas que respondieron el Inventario de creencias acerca de la violencia hacia la esposa, el Inventario de conflictos en las relaciones de noviazgo, y un cuestionario de información sociodemográfica. Los datos recolectados fueron examinados por medio de un análisis de correlación canónica, y los resultados mostraron que el modelo en general fue estadísticamente significativo (Wilks $\lambda=.654, F(20,677.54)=4.626, p<.05)$; que el tamaño del efecto del modelo general fue de .346 , lo que indica que este explicó el $34.6 \%$ de la varianza compartida por los dos conjuntos de variables; y que, específicamente en la primera función, el coeficiente de mayor magnitud fue el de la variable de justificación de la violencia $\left(r_{\mathrm{s}}^{2}=76.2 ; h^{2}=90.0\right)$, seguido por la del apoyo que se le puede brindar a la víctima $\left(r_{\mathrm{s}}^{2}=57.1 ; h^{2}=94.5\right)$.

Palabras clave: violencia en el noviazgo, creencias, justificación de la violencia, apoyo a la víctima, castigo al perpetrador.
\end{abstract}

Centro Universitario uaem, Josafat Pichardo \# 200. Col. Rancho la Mora, Toluca, México. Tel: 722-350-3968. Correo electrónico: dr.enrique.orozco@hotmail.com 


\title{
Beliefs about violence and its effects on the prevalence of dating violence
}

\begin{abstract}
Dating violence is a multifactorial phenomenon that requires in-depth studies regarding the effects that cultural beliefs have on the behavior of both the aggressor and the victim. With this in mind, the objective of this research was to determine the relationship between beliefs about violence and the prevalence of dating violence. To this end, a sample of 420 students from two Mexican public universities answered the Inventory of Beliefs about Wife Violence, the Inventory of Conflicts in Dating Relationships, and a sociodemographic information questionnaire. The data collected were examined through a canonical correlation analysis, and the results showed that the overall model was statistically significant (Wilks $\lambda=.654, F(20,677.54)=$ $4.626, p<.05$ ); that the effect size of the overall model was .346 , indicating that it explained $34.6 \%$ of the variance shared by the two sets of variables; and that, specifically in the first function, the coefficient of greatest magnitude was that of the variable of justification of the violence $\left(r^{2}=76.2 ; h^{2}=90.0\right)$, followed by that of the support that can be given to the victim $\left(r_{\mathrm{s}}^{2}=57.1 ; h^{2}=94.5\right)$.

Keywords: dating violence, beliefs, justification of violence, support for the victim, punishment of the perpetrator.
\end{abstract}

\section{Introducción}

La violencia en el noviazgo constituye un problema de salud pública tanto en países industrializados como en aquellos en vías de desarrollo, tanto en países con niveles altos de educación como en países con profundas carencias educativas, y tanto en países con servicios de salud adecuados como en países con una severa escasez de recursos en la atención médica y psicológica. De modo que, aun cuando algunos países gozan de los bienes y servicios adecuados, la violencia en contra de las mujeres no ha podido ser erradicada, e, incluso, en Latinoamérica la prevalencia de violencia en el noviazgo ha alcanzado índices preocupantes.

Por ejemplo, en una investigación llevada a cabo en Colombia con estudiantes de universidades públicas, la prevalencia de la violencia en el noviazgo fue del $85.6 \%$, y en sus resultados no se encontró una diferencia significativa entre hombres y mujeres, y sobresale la violencia de tipo psicológica como la más frecuente, seguida de la física, la emocional, la sexual, la económica y, finalmente, la negligencia (Rey-Anacona, 2013).

En México, por otra parte, los datos oficiales señalan que, aunque para el año 2008 solo el $15 \%$ de los jóvenes había sufrido de violencia física y un $16.5 \%$ había sido objeto de violencia sexual, el $76 \%$ había sufrido algún acto de violencia psicológica. Además, al preguntarle a los perpetradores, tanto hombres como mujeres, las razones que los motivaron a cometer diversos actos de violencia contra su pareja, los hombres señalaron que el principal motivo era los celos (41\%), seguido del número de amigos que tiene su pareja $(25.7 \%)$, y las mujeres afirmaron que la principal razón era también los celos (46\%), seguida de cuando su pareja se compromete a algo y no lo cumple $(42.5 \%) \mathrm{y}$, finalmente, por engaños (35\%) (Instituto Mexicano de la Juventud, 2008).

Asimismo, en un estudio realizado en estudiantes adolescentes de escuelas públicas en México, se encontró que la prevalencia de los actos violentos cometidos contra su novio o novia de tipo psicológico era de $4.3 \%$ para los hombres y de $4.2 \%$ para las mujeres, y que la prevalencia de la violencia física cometida en contra de la pareja aumentaba hasta el $20.9 \%$ en el caso de las mujeres, y hasta el $19.5 \%$ para los hombres. Al unir ambos tipos de violencia, los resultados indicaron que el $7.5 \%$ de las mujeres reportaron haber cometido actos violentos, en comparación con el $5.5 \%$ de los hombres (Rivera et al., 2007).

Ahora bien, un aspecto que cobra una importante relevancia respecto a la prevalencia de la violencia de pareja es el de las creencias y los procesos cognitivos de las personas involucradas en dicha problemática. Específicamente, existen creencias religiosas, culturales, políticas, morales, etc., pero, en esencia, una creencia se puede definir como un asentimiento cognitivo o emocional de un concepto, una proposición, una situación o una doctrina (Thompson, 1992), o como el conjunto de principios ideológicos o simples ideas que tiene una persona o un grupo social (Furinghetti $\&$ Pehkonen, 2002). No obstante, sabemos que, además de servirnos para tomar una decisión, intercambiar opiniones, formarnos una ideología y asumir una postura frente a un acontecimiento, las creencias son elementos determinantes en el comportamiento humano.

Ahora bien, como es de suponer, las creencias que favorecen o inhiben la conducta violenta, como sucede con las creencias patriarcales, el sexismo y las creencias de los roles tradicionales de género, que tienen efectos directos e indirectos en la prevalencia de distintos tipos de violencia hacia las mujeres. De hecho, considerada como la creencia 
156

de mayor impacto en la violencia de pareja, la ideología patriarcal ha influenciado radicalmente las relaciones interpersonales entre hombres y mujeres (Russo \& Pirlott, 2006), y se ha encontrado que en las sociedades consideradas como altamente patriarcales los hombres son más propensos a abusar de las mujeres, lo cual se ha convertido no solo en una práctica común, sino que también forma parte de sus creencias patriarcales (Tonsing \& Tonsing, 2019).

En términos generales, las creencias patriarcales no solo han subordinado el rol de la mujer, al conferirle la realización de tareas muy específicas dentro y fuera del hogar, sino que también han acentuado el control que el hombre tiene sobre las mujeres (Hunnicutt, 2009). Incluso, en las sociedades altamente patriarcales se presentan, principalmente entre los hombres, altos niveles de creencias en favor de la violencia contra las mujeres, así como estereotipos que desvalorizan el rol de la mujer en la sociedad (Hargreaves et al., 2006).

Como consecuencia del predominio del hombre, ha sido posible justificar la violencia contra las mujeres cuando estas trasgreden las normas socialmente establecidas (Tang et al., 2002). De esta manera, algunas situaciones, como la infidelidad de la mujer, el fallar en la crianza de los hijos, la búsqueda de independencia económica, y el deseo de divorciarse o separarse de su marido, son consideradas como razones suficientes para agredir a una mujer. Asimismo, se ha identificado que precarias condiciones socioeconómicas, como el tener una condición de salud deficiente o un nivel muy bajo de educación, son factores que inciden en la justificación de la violencia por parte de los hombres (Darteh et al., 2020).

Ahora bien, en la literatura científica sobre el tema se ha documentado la profunda relación que existe entre las creencias patriarcales y la prevalencia de la violencia de pareja. Por ejemplo, después de un extenso análisis de los resultados encontrados en más de sesenta investigaciones alrededor del mundo, Waltermaurer (2012) confirmó que las personas que tienen una gran adhesión a las creencias patriarcales tienden a justificar el uso de la violencia contra las mujeres, especialmente cuando juzgan que la mujer ha fallado en sus responsabilidades. Incluso, en otro estudio se encontró que la influencia de las creencias patriarcales es decisiva, en lo general, en las desigualdades de género que se presentan en la sociedad, así como, en lo particular, en las actitudes y comportamientos violentos hacia las mujeres (Sugarman \& Frankel, 1996).

Además de la ideología patriarcal, otro de los tipos de creencias que más se han investigado es el constructo de "sexismo", pues este tiene un impacto muy importante en la prevalencia de la violencia de pareja. De acuerdo con Ferrer-Pérez et al. (2006), el sexismo es una actitud adquirida que genera una respuesta evaluativa, afectiva, cognitiva y conductual hacia una persona en virtud de la pertenencia a su sexo biológico; $y$, entre las principales teorías que explican las creencias sexistas se encuentra la postulada por Glick y Fiske (1996), en la cual se afirma que el sexismo tiene una condición ambivalente, de la que se desprenden dos componentes rotundamente diferenciados: el sexismo hostil, que está relacionado con la creencia de que la mujer no solo debería ser controlada limitándola al ejercicio de roles de género muy específicos, sino que también es forzada a ser sumisa; y el sexismo benévolo, que enfatiza los estereotipos de género tradicionales. Desde esta teoría, cuando una mujer no se apega a aquellos estereotipos, el sexismo legitima inminentemente el uso de reacciones negativas, incluyendo la violencia contra las mujeres. Así, siendo una evaluación distorsionada de la mujer, las creencias sexistas generarían un comportamiento discriminatorio y violento.

De hecho, en la literatura científica se ha documentado ampliamente la relación entre el sexismo y la prevalencia de la violencia de pareja (p. ej., Abrams et al., 2003; Cava et al., 2020; Díaz-Aguado, 2003; Moya et al., 2007; Sakalli-Ugurlu et al., 2007). Por ejemplo, en un estudio con víctimas de violencia en el noviazgo y en el matrimonio, los resultados mostraron que el sexismo hostil estuvo asociado con la justificación del abuso sexual y con las actitudes negativas hacia las víctimas de abuso sexual (Durán et al., 2010); y, de la misma manera, en otra investigación — que tuvo como objetivo conocer las percepciones que se tienen ante una traición en el noviazgo-, Forbes et al. (2005) encontraron que los participantes justificaban el uso de la violencia cuando su pareja los engañaba.

Al comparar ambos sexos, Lameiras y Rodríguez (2002) identificaron que los hombres tienen más creencias sexistas y son más benévolos hacia los mismos hombres que hacia las mujeres; y en otra investigación las mujeres reportaron más creencias igualitarias en lo referente a la toma de decisiones en la familia, las actividades del hogar y la participación equitativa en la vida pública que los hombres (Díaz-Aguado, 2003). Recientemente, un estudio que examinó los efectos de las actitudes sexistas en la violencia en el noviazgo reveló que, en comparación con las mujeres, los hombres no solo reportaron niveles más altos en el sexismo benévolo y en el hostil, sino que la adherencia al sexismo también estaba fundamentada en patrones tradicionalmente patriarcales (Fernández-Antelo et al., 2020).

Por otra parte, con respecto a las creencias de los roles tradicionales de género, se ha considerado que estas son un factor de riesgo en las relaciones de noviazgo (Reitzel-Jaffe y Wolfe, 2001), debido a que la mayoría de los noviazgos ocurren durante la adolescencia, y que tanto hombres como mujeres tienden a intensificar en esta etapa las expectativas que tienen de su pareja, lo que resulta de gran importancia 
debido a que es bastante común que estas expectativas se basen en los roles tradicionales que enfatizan el control del hombre y la sumisión de la mujer. Además, si a esta condición se le añade la idealización romántica de la pareja, es posible explicar por qué algunas adolescentes aceptan el comportamiento controlador y violento de su novio. De acuerdo con Cauffman et al. (2000), este conjunto de creencias es determinante en el desarrollo de la violencia en el noviazgo.

Finalmente, se ha encontrado que el origen de la adquisición y aceptación de las creencias de los roles tradicionales de género se encuentra en la familia de origen, pues los adolescentes que han sido testigos de la violencia entre sus padres y hermanos, o quienes han experimentado actos violentos en su contra al estar viviendo con los miembros de su familiar, son más propensos a justificar el uso de la violencia en las relaciones con sus parejas. De hecho, en un estudio con adolescentes que habían visto actos violentos entre sus padres, los resultados mostraron que las mujeres tendían a aceptar más fácilmente la violencia en el noviazgo (Foshee et al., 1999).

Ahora bien, con base en el impacto que tienen las creencias familiares y culturales en la violencia, y siendo México un país con una fuerte presencia de creencias patriarcales, sexistas y tradicionales hacia los roles de género, el objetivo de la presente investigación fue determinar la relación entre las creencias hacia la violencia y la prevalencia de la violencia en el noviazgo, y como propósito general se tuvo identificar el grado de influencia que tienen las creencias culturales en el comportamiento tanto del agresor como de la víctima.

Derivado de ello, el estudio contó con dos hipótesis base: la primera, "existe una relación estadísticamente significativa entre las creencias hacia la violencia y la prevalencia de la violencia en el noviazgo" ( $\mathrm{H} 1)$; y la segunda, teniendo en cuenta que una de las creencias más relevantes en la violencia de pareja es la justificación de los actos cometidos por el agresor - donde, al justificar su comportamiento, el agresor considera que tiene el "derecho" de ejercer la violencia contra su pareja-, y que muchas víctimas justifican que sean abusadas al creer que ellas "se lo ganaron", se formuló que "la justificación del comportamiento violento es percibida como la creencia más importante en comparación con las ganancias-beneficios que puede obtener la víctima, el apoyo que se les brinda a las víctimas, el castigo hacia el agresor y la responsabilidad del agresor" ( $\mathrm{H} 2$ ). Con el fin de probar estas hipótesis, se planteó el análisis de correlación canónica para examinar el efecto de estas creencias en la violencia sexual, la violencia emocional, la violencia física y las amenazas hacia las víctimas.

\section{Método}

\section{Tipo de estudio}

La presente investigación es de corte cuantitativo, transversal y no experimental, y tiene un diseño correlacionalexplicativo, por medio del cual se analizaron los efectos de las creencias acerca de la violencia en la prevalencia de la violencia en el noviazgo (Creswell \& Creswell, 2018).

\section{Participantes}

Se obtuvo una muestra de 420 estudiantes de dos universidades públicas del Estado de México por medio de un muestreo no probabilístico e intencional. En la Tabla 1 se encuentran las características sociodemográficas de los estudiantes que hicieron parte del estudio.

\section{Instrumentos}

Inicialmente, se creó un cuestionario que incluyó diversas preguntas con la finalidad de recabar información sociodemográfica de los participantes, como su edad, sexo, el tiempo que llevaban en su relación romántica, el número de parejas que han tenido, el grado académico en el que se encontraban, y la licenciatura que estaban estudiando; y, posteriormente, se utilizó el "Inventario de creencias acerca de la violencia hacia la esposa" (Inventory of Beliefs About Wife Beating Scale) de Saunders et al. (1987) con el fin de evaluar las creencias acerca de la violencia hacia la mujer; por último, la violencia en el noviazgo se midió por medio del "Inventario de conflictos en las relaciones de noviazgo" (Conflict in Adolescent Dating Relationships Inventory) de Wolfe et al. (2001).

\section{Inventario de creencias acerca de la violencia hacia} la esposa

Esta escala, creada por Saunders et al. (1987) cuenta con un total de 32 reactivos agrupados cinco dimensiones: (a) justificación de la violencia, (b) ganancias-consecuencias de la violencia, (c) apoyo a la víctima, (d) castigo al agresor y (e) responsabilidad del agresor. Y, debido a que este instrumento sería utilizado para medir la relación en el noviazgo, se hizo una adaptación en la que se cambió la palabra "esposa" por "novia", así como "esposo" por "novio". De la misma manera, se sustituyó la palabra "matrimonio" por la palabra "noviazgo".

Específicamente, el primer factor, justificación de la violencia, cuenta con 12 reactivos que enumeran una serie de justificaciones empleadas comúnmente por el agresor y por aquellos que aprueban el uso de la violencia en contra de las mujeres - p. ej., "Ocasionalmente la violencia que 
ejerce un hombre en contra de su pareja puede ayudar a mantener su noviazgo"-

El segundo factor, ganancias-beneficios de la violencia (Wives gain from beatings), como lo refleja su nombre en inglés, enfatiza las posibles ganancias que obtiene una mujer al ser golpeada por su pareja, pues, como mencionan los autores de esta escala, en algunas situaciones, algunas mujeres presentan una experiencia placentera o masoquista al ser abusadas por su pareja (Saunders et al., 1987). Con base en ello, y con el fin de medir las creencias que enfatizan la "responsabilidad-culpabilidad" de la mujer en su propio abuso, los autores crearon esta dimensión, en la cual se encuentran reactivos como "Las novias que son golpeadas son responsables de ello porque ellas lo provocaron".

El tercer factor, apoyo a la víctima, incluye cinco reactivos que tienen el fin de identificar las creencias que llevan a una persona o institución a proveer de la protección y ayuda que requiere cualquier víctima de violencia - p. ej., "Si escucho que una mujer está siendo atacada por su novio, llamaría a la policía"-.

El cuarto factor, castigo al agresor, está constituido por cinco reactivos que corresponden a una serie de afirmaciones que destacan la necesidad de abandonar a la pareja cuando él agrede a su novia, así como el castigo que debería recibir una persona que agrede a su pareja - p. ej., "Si una novia es golpeada por su novio, ella debería separarse de él inmediatamente"-.

Y, por último, el quinto factor, responsabilidad del agresor, contiene dos reactivos que buscan medir aquellas creencias que señalan al agresor como el principal responsable de la violencia que se ejerce en contra de las víctimas - p. ej.. "Los novios quienes golpean a sus novias deberían ser responsables por el maltrato porque ellos debieron haber previsto que eso sucedería"-.

En general, la escala se contesta por medio de una escala tipo Likert de siete opciones (de $1=$ "Totalmente de acuerdo" a 7 = "Totalmente en desacuerdo"), y, la misma ha reportado niveles adecuados de confiabilidad interna con alfas que oscilan entre .61 y .91 (Haj-Yahia \& Uysal, 2008; Saunders et al.,1987). En la presente investigación se encontraron resultados similares en las puntaciones alfa de Cronbach de todas las dimensiones: justificación $(\alpha=.90)$, ganancias-beneficios $(\alpha=.71)$, apoyo a la víctima $(\alpha=.83)$, castigo al agresor $(\alpha=.84)$, y responsabilidad del agresor $(\alpha=.81)$.

Inventario de conflictos en las relaciones de noviazgo

Este instrumento, desarrollado por Wolfe et al. (2001), utilizado con el fin de medir la violencia en el noviazgo, está dividido en dos subescalas - violencia cometida y violencia sufrida - que cuentan con un total de 25 reactivos en cada una. Específicamente, esta escala consta de cinco dimensiones, a saber, violencia sexual, violencia relacional, violencia verbal-emocional, amenazas, y violencia física, cada una con buenos niveles de confiabilidad $-\alpha=.51$; $\alpha=.52 ; \alpha=.82 ; \alpha=.66 ; \mathrm{y} \alpha=.83$, respectivamente (Wolfe et al., 2001) -; cuyas opciones de respuesta se encuentran en un formato tipo Likert de cuatro opciones (de $0=$ "Nunca" a 3 = "Con frecuencia").

En esta investigación se determinó no incluir la subescala de violencia relacional, porque se quería medir específicamente la prevalencia de los tres tipos de violencia más reportados: la violencia psicológica, la física y la sexual. Además, al llevar a cabo el análisis de confiabilidad, se encontraron resultados similares para las cuatro dimensiones evaluadas: violencia sexual $(\alpha=.55)$, violencia verbal-emocional $(\alpha=.77)$, amenazas $(\alpha=.60)$, y violencia física $(\alpha=.88)$.

Finalmente, es importante mencionar que en la presente investigación se empleó la adaptación y versión al castellano desarrollada por Fernández-Fuertes et al. (2006). Estos autores analizaron la validez y fiabilidad de la escala y encontraron resultados parecidos a los publicados originalmente.

\section{Procedimiento}

Después de haber obtenido por parte de los directivos de ambas universidades la autorización para llevar a cabo esta investigación, se elaboró la estrategia correspondiente para recolectar los datos. El presente estudio cumplió con todos los requisitos y normas éticas que se han establecido en México para la recolección y el análisis de los datos en seres humanos, de manera que, antes de contestar los instrumentos, los participantes otorgaron su consentimiento, y solo aquellos que voluntariamente decidieron participar respondieron las dos escalas.

Para la aplicación de los instrumentos, en un primer momento se les explicó a los alumnos el objetivo de la investigación, las características de esta y sus derechos como participantes. Además, se enfatizó el aspecto voluntario y anónimo del estudio, y que en cualquier momento podían decidir no continuar respondiendo los instrumentos. La recolección de datos se hizo de manera grupal, con un promedio de duración de 20 minutos.

\section{Análisis de datos}

Inicialmente, se realizó un análisis descriptivo de los reactivos del instrumento de creencias con el propósito de identificar los reactivos con los cuales los participantes estaban más de acuerdo; posteriormente, se analizaron 
diferentes correlaciones bivariadas entre las variables sociodemográficas y las variables de estudio; y, por último, se llevó a cabo el análisis de correlación canónica con la finalidad de examinar la hipótesis medular.

De acuerdo con Sherry y Henson (2005), el análisis de correlación canónica es una prueba multivariante apropiada cuando el propósito de una investigación es examinar la relación que pudiese existir entre dos conjuntos de variables. Entre las principales ventajas de utilizar el análisis de correlación canónica se encuentra, por una parte, la posibilidad de reducir el riesgo de cometer un error de Tipo I (Thompson, 1991); y, por otra, que, al utilizar una técnica multivariante como el análisis de correlación canónica, el investigador es capaz de examinar el vínculo que se presenta entre múltiples causas y múltiples efectos. Asimismo, a diferencia de otros análisis en los cuales solo se tiene la posibilidad de analizar el efecto de múltiples factores en una sola variable - p. ej. regresión múltipleel análisis de correlación canónica permite profundizar en el conocimiento de la complejidad de ciertos fenómenos psicológicos (Fish, 1988).

Finalmente, siguiendo las recomendaciones de Hair et al. (1984), se estableció el nivel de significancia en .05, por considerarse el más apropiado cuando se efectúa un análisis de correlación canónica.

\section{Resultados}

Como se puede observar, la dispersión y los porcentajes de los datos sociodemográficos de los participantes de la muestra se encuentran en la Tabla 1 , y los estadísticos descriptivos de cada una de las dimensiones correspondientes a las variables del estudio aparecen en la Tabla 2.

Tabla 1.

Datos sociodemográficos

\begin{tabular}{lcccc}
\hline \multicolumn{1}{c}{ Variable } & $\%$ & Variable & $M$ & $D T$ \\
\hline Sexo & & Edad & 21.04 & 1.65 \\
$\quad$ Mujeres & 51.4 & Años de noviazgo & 1.8 & 1.2 \\
$\quad$ Hombres & 48.6 & & \\
Semestre & & & \\
$2^{\circ}$ & 4.2 & & \\
$4^{\circ}$ & 24.8 & & \\
$6^{\circ}$ & 30.4 & & \\
$8^{\circ}$ & 40.6 & & \\
Carrera & & & \\
Administrativas & 18.3 & & \\
Ingeniería & 45.8 & & \\
Ciencias sociales & 35.9 & & \\
\hline
\end{tabular}

Tabla 2.

Media y desviación típica de las variables de estudio

\begin{tabular}{lcc}
\hline \multicolumn{1}{c}{ Variable } & $M$ & $D T$ \\
\hline Justificación & 21.72 & 9.50 \\
Ganancias-beneficios & 11.57 & 5.55 \\
Apoyo a la víctima & 25.58 & 4.47 \\
Castigo al agresor & 17.61 & 4.56 \\
Responsabilidad del agresor & 8.02 & 2.85 \\
Violencia física & 2.03 & 1.02 \\
Violencia emocional & 9.89 & 2.61 \\
Violencia sexual & 4.92 & 3.53 \\
Amenazas & 1.10 & 0.87 \\
\hline
\end{tabular}

Con la finalidad de conocer específicamente las creencias que prevalecen en los hombres, así como en las mujeres, se analizaron por separado las respuestas de ambos grupos, con lo cual se encontró que, en lo concerniente a las creencias de los hombres, el $85.3 \%$ estuvo totalmente de acuerdo o de acuerdo con la afirmación "Las organizaciones sociales deberían hacer más por ayudar a las mujeres maltratada" $(M=2.15, D T=1.32)$. Esta afirmación corresponde a la dimensión de apoyo a la víctima, y obtuvo el porcentaje más elevado entre los hombres, seguida por la afirmación "Una novia debería irse de la relación si su novio la golpea" $(M=3.58, D T=1.67)$, correspondiente a la dimensión de castigo al agresor, con el $62.5 \%$ de asentimiento.

Con respecto a la dimensión de las ganancias-beneficios de la violencia, la afirmación con la que los hombres más estuvieron de acuerdo o totalmente de acuerdo fue "Las novias quienes son golpeadas son responsables de ello porque ellas debieron haber previsto que eso sucedería" $(M=6.65, D T=1.22)$; no obstante, esto solo ocurrió en el $6.9 \%$ de la muestra. Finalmente, en la dimensión que corresponde a la justificación de la violencia, la afirmación que tuvo el mayor número de respuestas aprobatorias fue "Una novia infiel sexualmente merece ser golpeada" $(M=2.77, D T=1.94)$, pues el $25.3 \%$ de los hombres estuvo de acuerdo o totalmente de acuerdo con ella.

Por otra parte, la afirmación con la que las mujeres estuvieron más de acuerdo o totalmente de acuerdo fue "Las mujeres deberían ser protegidas por las leyes si sus novios las golpean" $(M=1.53, D T=.94)$, correspondiente a la dimensión de apoyo a la víctima, pues el $90.3 \%$ de las mujeres manifestaron su aprobación con esta afirmación. Con respecto a la cuarta dimensión, la afirmación que tuvo el porcentaje más alto de asentimiento $(73.7 \%)$ fue "Si una novia es golpeada por su novio, ella debería separarse de él inmediatamente" $(M=2.99, D T=1.30)$; a su vez, en la dimensión que corresponde a las ganancias-beneficios de la violencia, la afirmación que tuvo el mayor grado de 
160

acuerdo fue "Las mujeres sienten dolor y no placer cuando son golpeadas por su novio" $(M=2.01, D T=1.79)$, con un $81.5 \%$ de aprobación; $y$, finalmente, para la dimensión de justificación de la violencia, la afirmación con la que las participantes estuvieron más de acuerdo o totalmente de acuerdo (16.5\%) fue "Una novia infiel sexualmente merece ser golpeada" $(M=2.13, D T=1.80)$.

Posteriormente, se examinaron los coeficientes de correlación de Pearson entre las dimensiones de las variables de creencias y las de violencia en el noviazgo, antes de llevar a cabo el análisis de correlación canónica (véase Tabla 3).

Teniendo en cuenta que el objetivo central de esta investigación fue analizar la relación existente entre las creencias hacia la violencia - específicamente, las creencias de la justificación de la violencia, las ganancias-beneficios que pudiese obtener la víctima, el apoyo hacia las víctimas, el castigo del agresor y la responsabilidad del agresor-y la prevalencia de la violencia - a saber, los factores de violencia sexual, emocional y física, así como las amenazas que reciben las víctimas - que se presenta en parejas de novios; para el análisis de los datos se estableció que los cinco factores de las creencias acerca de la violencia fueran los predictores de las cuatro dimensiones que conforman la prevalencia de la violencia en el noviazgo. De esta manera, se examinó la relación multivariante que comparten estos dos conjuntos de variables.

Antes de llevar a cabo el análisis de correlación canónica se verificaron distintos supuestos siguiendo la propuesta hecha por Tabachnick y Fidell (2012), con lo cual se encontró que, con respecto al supuesto de normalidad — validado por medio de los coeficientes de asimetría y curtosis-, todas las variables de estudio mostraron valores entre -1 y 1 , lo que da cuenta de la distribución normal de los datos; así como que, con respecto al supuesto de homocedasticidad - verificado por medio de las distribuciones de los residuales-, los resultados mostraron que la variación de los residuos fue uniforme en todos los gráficos, por lo que no se encontraron pautas de asociación; $y$, por último, para el supuesto de linealidad - donde se correlacionaron en pares todas las dimensiones de las creencias hacia la violencia con las de la violencia en el noviazgo - , que la gran mayoría de las dimensiones de las creencias correlacionaron de manera significativa con las de la violencia - con excepción de la dimensión de responsabilidad. Finalmente, en la matriz de correlación no se identificó el problema de la multicolinealidad $(r>.90)$.

Ahora bien, con respecto a los resultados del análisis de correlación canónica, en general se encontró que el modelo en general fue estadísticamente significativo (Wilks $\lambda=.654, F(20,677.54)=4.626, p<.05)$, lo cual indica que hay una correlación significativa entre el conjunto de las creencias hacia la violencia y el conjunto de los tipos de violencia en el noviazgo.

Específicamente, el criterio de Wilks $\lambda$ refleja la varianza que no es explicada por el modelo, a la vez que $1-\lambda$ representa el tamaño del efecto del modelo completo en una $r^{2}$ métrica; de esta manera, el tamaño del efecto $r^{2}$ del modelo general fue de .346 , lo cual indica que el modelo en general explicó el $34.6 \%$ de la varianza compartida por los dos conjuntos de variables.

Una vez que el modelo canónico es significativo, es necesario analizar cada una de las funciones canónicas - en este caso, las cuatro funciones- $-\mathrm{y}$, como se puede observar en la Tabla 4, la correlación canónica al cuadrado para la función 1 fue de .248 , mientras que para la función 2 fue de .113, para la función 3 fue de .018, y, para la última función, de .002. Con respecto a la distribución jerárquica de las cuatro funciones del modelo, las funciones 1 y 2 - que comprenden el modelo en general- fueron estadísticamente significativas.

Como se muestra en la Tabla 4, el modelo en general, así como las dos primeras funciones, fueron estadísticamente

Tabla 3.

Matriz de correlaciones bivariadas

\begin{tabular}{lccccccccc}
\hline \multicolumn{1}{c}{ Variable } & 1 & 2 & 3 & 4 & 5 & 6 & 7 & 8 & 9 \\
\hline 1. Justificación & - & & & & & & & & \\
2. Ganancias & $.56^{* *}$ & - & & & & & & \\
3. Apoyo & $-.36^{* *}$ & $-.38^{* *}$ & - & & & & & \\
4. Castigo & $-.23^{* *}$ & -.18 & $.59^{* *}$ & - & & & & \\
5. Responsabilidad & -.11 & -.09 & $.36^{* *}$ & $.52^{* *}$ & - & & & \\
6. Violencia sexual & $.34^{* *}$ & $.22^{* *}$ & $-.33^{* *}$ & $-.17^{*}$ & -.07 & - & & \\
7. Violencia emocional & $.40^{* *}$ & $.18^{* *}$ & $-.14^{* *}$ & .02 & .04 & $.62^{* *}$ & - & \\
8. Violencia física & $.38^{* *}$ & $.24^{* *}$ & $-.40^{* *}$ & $-.22^{* *}$ & -.10 & $.71^{* *}$ & $.56^{* *}$ & - & - \\
9. Amenazas & $.30^{* *}$ & .12 & $-.22^{* *}$ & -.08 & .02 & $.68^{* *}$ & $.69^{* *}$ & $.72^{* *}$ & - \\
\hline
\end{tabular}

Nota. $p<.05$. 
Tabla 4.

Análisis de correlación canónica de los conjuntos dependiente e independiente

\begin{tabular}{|c|c|c|c|c|}
\hline Función canónica & Correlación canónica & $R^{2}$ canónica & $F$ & Probabilidad \\
\hline \multicolumn{5}{|c|}{ Medidas de ajuste del modelo general para correlación canónica } \\
\hline 1 & .4985 & .248 & 4.62 & .000 \\
\hline 2 & .3368 & .113 & 2.43 & .004 \\
\hline 3 & .1338 & .018 & 0.63 & .705 \\
\hline 4 & .0155 & .002 & 0.03 & .976 \\
\hline \multicolumn{2}{|c|}{ Pruebas multivariantes de significancia } & Valor & Aprox. $F$ & Probabilidad \\
\hline \multicolumn{2}{|c|}{ Pillais } & .380 & 4.348 & .000 \\
\hline \multicolumn{2}{|c|}{ Hotellings } & .477 & 4.832 & .000 \\
\hline \multicolumn{2}{|c|}{ Wilks } & .654 & 4.626 & .000 \\
\hline \multicolumn{2}{|c|}{ Roys } & .248 & & \\
\hline
\end{tabular}

significativas. Ahora, en el análisis de correlación canónica se presentan tantas funciones como variables se encuentran en el conjunto más pequeño de variables, por lo que, en el caso particular de esta investigación, en el conjunto de predictores hay cinco variables, mientras que en el conjunto de las variables de criterio se incluyen cuatro variables; no obstante, de la misma manera en que se emplea en el análisis factorial, solo se definen e interpretan aquellas funciones que explican una cantidad razonable de la relación que guardan entre sí el conjunto de predictores y el conjunto de variables de criterio (Sherry \& Henson, 2005).

Por otra parte, cada función canónica contiene un conjunto de coeficientes canónicos estandarizados y cada función es ortogonal a todas las otras funciones que se presentan en un diseño de investigación; de esta manera, cada conjunto de variables predictoras y de criterio sintéticas no se encontrarán perfectamente correlacionadas con todas las otras variables predictoras y de criterio sintéticas presentes en otras funciones. Debido a esta ortogonalidad, las funciones en el análisis de correlación canónica son semejantes a los componentes que se encuentran en un análisis principal de componentes. Así, comparándolo con un análisis de regresión múltiple, cada función es similar al conjunto de pesos de regresión estandarizados de los predictores (Tabachnick \& Fidell, 2012; Thompson, 1984).

En el presente estudio, los efectos de estas dos funciones explicaron una cantidad estadísticamente significativa de la varianza compartida entre los dos conjuntos de variables. Específicamente, la función 1 explicó el $24.8 \%$ de varianza compartida, la función 2 explicó el $11.3 \%$ de varianza compartida, y las otras funciones ( 3 y 4 ) solo explicaron el $2.0 \%$ de la varianza que quedaba después de la extracción de las primeras dos funciones.

Por otra parte, en la Tabla 5 se encuentran los coeficientes estandarizados, así como los coeficientes de la estructura que se obtuvieron en las funciones 1 y 2 , y los coeficientes de comunalidad de cada una de las variables tanto de criterio como los predictores. De acuerdo con Temurtas (2016), los coeficientes de estructura y los valores mayores a .45 presentan una contribución estadísticamente significativa a la función, al igual que los coeficientes de comunalidad mayores a .45 , pues tienen una contribución estadísticamente significativa al modelo. En esta tabla se presentan los coeficientes de las dos primeras funciones canónicas con la finalidad de poder compararlos, pues los coeficientes de la función canónica estandarizada proporcionan la contribución de cada uno de los conjuntos de las variables a las funciones canónicas, y son estos los que se usan para estimar los valores de las características analizadas al momento de llevar a cabo la investigación.

En este punto, los resultados muestran que, para la primera función, las variables de criterio más significativas fueron la violencia física y la violencia sexual, con una contribución secundaria de la violencia emocional y las amenazas. Con esta información, y con base en los coeficientes de estructura al cuadrado, así como en los coeficientes de la función canónica, se puede concluir que la violencia física presentó la magnitud más importante en las variables de criterio. Adicionalmente, con excepción de la variable de amenazas, los tres tipos de violencia tuvieron el mismo signo, lo cual indica que la violencia sexual, emocional y física están relacionadas positivamente.

Por otra parte, respecto a los cinco predictores en la primera función, destacan la justificación y el apoyo hacia las víctimas como los predictores de mayor impacto, mientras que, con una aportación secundaria, se encuentran las ganancias-beneficios que podría obtener una víctima de la violencia en el noviazgo. Este hallazgo avala el planteamiento hecho en la hipótesis central de esta investigación, de manera que se puede concluir que la justificación del comportamiento violento es percibida como la creencia más importante en comparación con las ganancias-beneficios 
Tabla 5.

Correlación canónica para las creencias como predictores de la violencia en el noviazgo para las funciones 1 y 2

\begin{tabular}{|c|c|c|c|c|c|c|c|}
\hline \multirow{2}{*}{ Variable } & \multicolumn{3}{|c|}{ Función 1} & \multicolumn{3}{|c|}{ Función 2} & \multirow[b]{2}{*}{$h^{2}(\%)$} \\
\hline & Coef. & $r_{\mathrm{s}}$ & $r_{\mathrm{s}}^{2}(\%)$ & Coef. & $r_{\mathrm{s}}$ & $r_{\mathrm{s}}^{2}(\%)$ & \\
\hline Violencia sexual & -.24 & -.81 & 66.1 & .49 & .08 & 0 & 66.1 \\
\hline Violencia emocional & -.37 & -.74 & 54.5 & -1.22 & -.66 & 43.3 & 97.8 \\
\hline Amenazas & .33 & -.66 & 43.9 & -.18 & -.19 & 3.7 & 47.6 \\
\hline Violencia física & -.80 & -.94 & 88.2 & .64 & .19 & 3.7 & 91.9 \\
\hline$R^{2}{ }_{\mathrm{c}}$ & & & 38.8 & & & 25.3 & \\
\hline Justificación & -.72 & -.87 & 76.2 & -.80 & -.37 & 13.8 & 90 \\
\hline Ganancias-beneficios & .06 & -.55 & 30.0 & .23 & .07 & 0 & 30 \\
\hline Apoyo & .60 & .76 & 57.1 & -.48 & -.61 & 37.4 & 94.5 \\
\hline Castigo & -.11 & .37 & 13.5 & -.52 & -.70 & 49.1 & 62.6 \\
\hline Responsabilidad & -.05 & .18 & 3.3 & -.06 & -.43 & 18.7 & 22 \\
\hline
\end{tabular}

Nota. Coef. $=$ Coeficiente de la función canónica estandarizada; $r^{2}=$ coeficiente de estructura; $r_{\mathrm{s}}^{2}=$ coeficiente de estructura al cuadrado; $h^{2}=$ coeficiente de comunalidad. Los coeficientes de estructura mayores a .45 y los coeficientes de comunalidad mayores al $45 \%$ están en negrilla.

que puede obtener la víctima, el apoyo que se les brinda a las víctimas, el castigo hacia el agresor y la responsabilidad del agresor. Por último, los resultados en la segunda función mostraron que solo la violencia emocional tuvo una aportación relevante; mientras que, con respecto a los predictores, el castigo al agresor y el apoyo hacia la víctima presentaron la mayor magnitud.

Finalmente, como resultado del análisis de correlación canónica en ambas funciones, es posible concluir que se obtuvo suficiente evidencia empírica para fundamentar la relación entre las creencias y la violencia en el noviazgo.

\section{Discusión}

Las creencias que las personas poseen con respecto a cualquier condición o fenómeno social influyen en su comportamiento. Específicamente, en la violencia de pareja, el impacto de las creencias es determinante en la conducta que tiene tanto el agresor como la víctima, tal como se demostró en el presente estudio, pues los hallazgos que se encontraron en las pruebas multivariantes de significancia (Pillais, Hotellings, Wilks y Roys) y en las funciones canónicas mostraron que el modelo general fue estadísticamente significativo, y que los efectos de los predictores en las variables de criterio enfatizan la importancia de analizar las creencias de los jóvenes con la finalidad de estimar su impacto en el comportamiento violento.

Asimismo, con base en los coeficientes de la función canónica estandarizada, se encontró que el coeficiente de mayor magnitud fue el de la variable de justificación de la violencia, pues los participantes que reportaron los niveles más altos de violencia sexual contra su pareja presentaron una mayor cantidad de creencias por medio de la cuales avalan el uso de la violencia (justificación). De hecho, la creencia de justificar la violencia está directamente asociada con las creencias tradicionales de los roles de género (Reitzel-Jaffe $\&$ Wolfe, 2001), pues se ha encontrado que las personas que creen que la mujer debe ocupar las funciones propias de la madre y ama de casa, mientras que el hombre debe ser el proveedor, tienden a justificar el uso de la violencia contra las mujeres cuando ellas no cumplen estas funciones sociales y familiares.

Respecto a esto último, en un estudio con estudiantes de una universidad pública en EE. UU. se encontró que uno de los principales factores de la violencia en el noviazgo es la adhesión a los estereotipos de los roles de género (Mahlstedt \& Welsh, 2005); y en otra investigación, los resultados mostraron que los participantes con creencias que consideraban a las mujeres como inferiores e indignas de respeto, aceptaban en mayor medida el uso de la violencia en contra de sus novias, particularmente si habían sido infieles (Forbes et al., 2005). Asimismo, este mismo tipo de creencias fueron empleadas por los participantes de otro estudio llevado a cabo en Shanghai, Taiwan y Hong Kong para justificar al agresor y el involucramiento en diversos actos violentos en contra de las mujeres (Shen et al., 2012).

Por otra parte, la teoría del aprendizaje social postula que la justificación de la violencia forma parte de uno de los ocho mecanismos que derivan en el comportamiento violento (Bandura et al.,1996). De acuerdo con esta teoría, la justificación moral es un mecanismo empleado por las personas para reinterpretar el daño que provocan a los otros, de tal forma que resulte moralmente justificable. En 
este mismo sentido, Bandura (1991) afirmó que alguien se justifica moralmente cuando separa sus principios éticos y morales de su comportamiento. En este caso, es más probable que la persona comience a racionalizar su conducta amoral con el fin de conseguir intereses personales o avalar su comportamiento. Por ejemplo, un novio puede justificar que él golpea a su pareja porque ella le fue infiel. La misma condición puede ser empleada en cualquier caso en el que se encuentren motivos suficientes para justificar diversas conductas.

En el caso particular de esta investigación, los participantes mencionaron que el ejercicio de la violencia es justificable principalmente por tres motivos: (a) cuando la mujer llega a ser infiel sexualmente, (b) cuando se niega a tener relaciones sexuales, y (c) cuando tiene un comportamiento que amerita el uso de la violencia en su contra. Aunque otro tipo de creencias fueron exclusivas de los hombres, en la mayoría de las creencias que justifican el uso de la violencia, incluyendo estas tres, tanto hombres como mujeres, por igual, estuvieron de acuerdo con ellas. Al respecto, es de destacar que los dos motivos principales están relacionados con la actividad sexual; particularmente, las creencias sexistas y patriarcales hacen mucho énfasis en señalar a la mujer como un "objeto" sexual a la disposición del hombre.

Ahora bien, al comparar el nivel de justificación de los hombres y el de las mujeres, los resultados mostraron que los hombres estuvieron más de acuerdo con las afirmaciones que justifican el uso de la violencia contra las mujeres; resultado que está en concordancia con varios estudios que han señalado repetidamente que los hombres tienden a justificar más los actos violentos en comparación con las mujeres (p. ej., Calvete \& Cardeñoso, 2005; Díaz-Aguado \& Martínez, 2001; Lewis \& Fremouw, 2001).

Por otra parte, con base en los coeficientes de la función canónica estandarizada, se identificó que el apoyo que se le puede brindar a la víctima fue el segundo factor que tuvo los coeficientes con mayor magnitud. Al respecto, es interesante observar que el $90.8 \%$ de los participantes manifestó estar totalmente de acuerdo o de acuerdo con las diversas afirmaciones que expresaban la necesidad e importancia de brindar ayuda a las víctimas de la violencia de pareja. Específicamente, empleando el Inventario de creencias acerca de la violencia hacia la esposa, los participantes expresaron no solo la posibilidad de ellos mismos dar el apoyo, sino también la responsabilidad que tiene la policía, las instituciones gubernamentales y las organizaciones civiles de hacerlo.

Podría resultar un tanto contradictorio que coexistan estas dos creencias, pues, por un lado, se mantiene el predominio del hombre, el apego a las normas tradicionales, la justificación de la violencia cuando la mujer quebranta las normas morales y el control que el hombre ejerce sobre los roles femeninos; y por otro, la creencia de que, cuando una mujer es abusada - generalmente una mujer con la cual no se tiene ningún vínculo familiar o social一, se le debe brindar la atención y el apoyo que requiere.

Al respecto, diversas investigaciones han documentado la importancia que tiene el apoyo social en la prevalencia de la violencia de pareja. Por ejemplo, en una investigación llevada a cabo recientemente, los resultados mostraron que el apoyo que se les brinda a las víctimas de la violencia de pareja es uno de los factores de protección más importantes que pueden recibir, pues no solo les permite conservar y desarrollar su propia identidad como mujeres, superar los traumas que trae consigo el abuso que se ejerció en contra de ellas, y encontrarle un sentido al dolor que han experimentado, sino que también el apoyo que proviene de sus familias, amigos e instituciones funciona como un recurso que mitiga los efectos negativos de la violencia (Žukauskienè et al., 2019).

Asimismo, Bybee y Sullivan (2002) encontraron que el apoyo social que recibe una mujer es decisivo para evitar la presencia de abusos en los primeros años de vida en pareja. $\mathrm{E}$ incluso, de acuerdo con Zink et al. (2003), se ha encontrado que la carencia de apoyo social, gubernamental y familiar hacia las víctimas de la violencia provoca que muchas mujeres que quieren abandonar a sus parejas desistan de hacerlo. De hecho, en un estudio longitudinal con mujeres afroamericanas que buscaban ayuda por haber sido abusadas por su pareja, los resultados mostraron que el apoyo social que recibieron fue determinante no solo para mejorar su salud emocional y física, sino también para sentirse protegidas de futuros ataques por parte del agresor (Goodman et al., 2005). De la misma manera, en otra investigación con mujeres hispanas que migraron a EE. UU. se encontró que el apoyo de la familia y la protección del gobierno fueron determinantes para que las víctimas pudieran abandonar al agresor (Orozco et al., 2012).

Finalmente, a pesar de las aportaciones de esta investigación, existen algunas limitaciones que deben considerarse. La primera de ellas está relacionada con el uso de los instrumentos de autorreporte, pues, siendo estudiantes universitarios los que conformaron la muestra, este tipo de instrumentos no está exento de las respuestas falsas que emiten los participantes, de la deseabilidad social, de las dificultades en recordar con exactitud las experiencias vividas y de la exageración al contestar algunos reactivos. La segunda limitación tiene que ver con la medición que se hace con el fenómeno de la violencia y las creencias que la fundamentan; por la complejidad que representa la violencia, es muy difícil que los participantes revelen con 
exactitud ya sea cuando han sido violentos con su pareja o cuando han sido agredidos por su pareja; por consiguiente, es difícil saber con exactitud si la prevalencia que están reportando es correcta. Lo mismo sucede con las creencias hacia la violencia, pues, aunque la información es anónima y confidencial, no todos los participantes mencionan verazmente que creen en el uso de la violencia en contra de su pareja romántica. Por último, esta investigación no incluyó variables de control que tuviesen relación directa con el ambiente familiar, por lo que, debido al gran impacto que tiene la familia en México, futuras investigaciones deberían incluir la medición de los factores familiares que inciden directamente en las creencias de los jóvenes y su posterior comportamiento.

\section{Referencias}

Abrams, D., Viki, G. T., Masser, B., \& Bohner, G. (2003). Perception of a stranger and acquaintance rape: The role of benevolent and hostile sexism in victim blame and rape proclivity. Journal of Personality and Social Psychology, 84(1), 111-125. http://dx.doi.org/10.1037/0022-3514.84.1.111

Bandura, A. (1991). Social cognitive theory of moral thought and action. En W. M. Kurtines y J. L. Gewirtz (Eds.), Handbook of moral behavior and development: Theory, research and applications (pp. 71-129). Erlbaum.

Bandura, A., Barbaranelli, C., Caprara, G. V., \& Pastorelli, C. (1996). Mechanisms of moral disengagement in the exercise of moral agency. Journal of Personality and Social Psychology, 71(2), 364-374. http://dx.doi. org/10.1037/0022-3514.71.2.364

Bybee, D. I., \& Sullivan, C. M. (2002). The process through which an advocacy intervention resulted in positive change for battered women over time. American Journal of Community Psychology, 30(1), 103-132. http://dx.doi. org/10.1023/A:1014376202459

Calvete, E., \& Cardeñoso, O. (2005). Gender differences in cognitive vulnerability to depression and behavior problems in adolescents. Journal of Abnormal Child Psychology, 33, 179-192. http://dx.doi.org/10.1007/s10802-005-1826-y

Cauffman, E., Feldman, S. S., Arnett, L. J., \& Jensen, J. A. (2000). The (un)acceptability of violence against peers and dates. Journal of Adolescent Research, 15(6), 652-673. http://dx.doi.org/10.1177/0743558400156003

Cava, M. J., Martínez-Ferrer, B., Buelga, S., \& Carrascosa, L. (2020). Sexist attitudes, romantic myths, and offline dating violence as predictors of cyber dating violence perpetration in adolescents. Computers in Human Behavior, 111, 106449. http://dx.doi.org/10.1016/j.chb.2020.106449

Creswell, J. W., \& Creswell, J. D. (2018). Research design: Qualitative, quantitative, and mixed methods approaches (5. ${ }^{\mathrm{a}}$ ed.). Sage publications.
Darteh, E. K. M., Dickson, K. S., Rominski, S. D., \& Moyer, C. A. (2020). Justification of physical intimate partner violence among men in sub-Saharan Africa: a multinational analysis of demographic and health survey data. Journal of Public Health, 33, 1-9. http://dx.doi.org/10.1007/ s10389-020-01260-9

Díaz-Aguado, M. J. (2003). Adolescencia, sexismo y violencia de género. Papeles del Psicólogo, 23(84), 35-44. http:// www.papelesdelpsicologo.es/resumen?pii=1053

Díaz-Aguado, M. J., \& Martínez, A. (2001). La construcción de la igualdad y la prevención de la violencia contra la mujer desde la educación secundaria. Instituto de la mujer.

Durán, M., Moya, M., Megías, J. L., \& Viki, G. T. (2010). Social perception of rape victims in dating and married relationships: the role of perpetrator's benevolent sexism. Sex Roles, 62, 505-519. http://dx.doi.org/10.1007/ s11199-009-9676-7

Fernández-Antelo, I., Cuadrado-Gordillo, I., \& Martín-Mora, G. (2020). Synergy between acceptance of violence and sexist attitudes as a dating violence risk factor. International Journal of Environmental Research and Public Health, 17(14), 5209. https://doi.org/10.3390/ijerph17145209

Fernández-Fuertes, A. A., Fuertes, A., \& Pulido, R. F. (2006). Evaluación de la violencia en las relaciones de pareja de los adolescentes. Validación del Conflict in Adolescent Dating Relationships Inventory (CADRI) - versión española. International Journal of Clinical and Health Psychology, 6(2), 339-358. https://dialnet.unirioja.es/servlet/articulo?co digo $=1983727 \&$ orden $=65963$ \&info $=$ link

Ferrer-Pérez, V. A., Bosch-Fiol, E., Ramis-Palmer, M. C., \& Navarro-Guzmán, C. (2006). Las creencias y actitudes sobre la violencia contra las mujeres en la pareja: Determinantes sociodemográficos, familiares y formativos. Anales de psicologia, 22(2), 251-259. http://www.redalyc.org/articulo. oa? id $=16722210$

Fish, L. (1988). Why multivariate methods are usually vital. Measurement and Evaluation in Counseling and Development, 21(3), 130-137. http://dx.doi.org/10.1080/07 481756.1988.12022895

Forbes, G. B., Jobe, R. L., White, K. B., Bloesch, E., \& AdamsCurtis, L. E. (2005). Perceptions of dating violence following a sexual or nonsexual betrayal of trust: effects of gender, sexism, acceptance of rape myths, and vengeance motivation. Sex Roles, 52(3-4), 165-173. https://doi.org/10.1007/ s11199-005-1292-6

Foshee, V. A., Bauman, K. E., \& Linder, G. F. (1999). Family violence and the perpetration of adolescent dating violence: Examining social learning and social control processes. Journal of Marriage and the Family, 61(2), 331-342. http:// dx.doi.org/10.2307/353752

Furinghetti, F., \& Pehkonen, E. (2002). Rethinking characterizations of beliefs. En G. C. Leder, E. Pehkonen y G. Törner (Eds.). Beliefs: A hidden variable in mathematics education? (pp. 39-57). Kluwer Academic Publishers. 
Glick, P., \& Fiske, S. T. (1996). The Ambivalent Sexism Inventory: Differentiating hostile and benevolent sexism. Journal of Personality and Social Psychology, 70(3), 491512. http://dx.doi.org/10.1037/0022-3514.70.3.491

Goodman, L., Dutton, M. A., Vankos, N., \& Weinfurt, K. (2005). Women's resources and use of strategies as risk and protective factors for reabuse over time. Violence Against Women, 11(3), 311-336. http://dx.doi. org/10.1177/1077801204273297

Hair, J. F., Anderson, R. E., Tatham, R. L., \& Black, W. C. (1984). Multivariate Data Analysis. Prentice Hall.

Haj-Yahia, M., \& Uysal, A. (2008). Beliefs about wife beating among medical students from Turkey. Journal of Family Violence, 23, 119-113. http://dx.doi.org/10.1007/ s10896-007-9134-7

Hargreaves, S., L. Vetten, V. Schneider, L. Malepe, L., \& Fuller, R. (2006). Marriage is like sitting on red coals: A case study of domestic violence in four villages of the Moretele District, Tshwane metropole. Center for the Study of Violence and Reconciliation.

Hunnicutt, G. (2009). Varieties of patriarchy and violence against women: Resurrecting "patriarchy" as a theoretical tool. Violence Against Women, 15(5), 553-573. http:// dx.doi.org/10.1177/1077801208331246

Instituto Mexicano de la Juventud. (2008). Encuesta nacional de violencia en las relaciones de noviazgo 2007. INEGI.

Lameiras, M., \& Rodríguez, Y. (2002). Evaluación del sexismo moderno en los adolescentes. Revista de Psicología Social, 17(2), 119-127. https://doi. org $/ 10.1174 / 021347402320007555$

Lewis, S. F., \& Fremouw, W. (2001). Dating violence: A critical review of the literature. Clinical Psychology Review, 21(1), 105-127. https://doi.org/10.1016/S0272-7358(99)00042-2

Mahlstedt, D. L., \& Welsh, L. A. (2005). Perceived causes of physical assault in heterosexual dating relationships. Violence Against Women, 11(4), 447-472. http://dx.doi. org/10.1177/1077801204273298

Moya, M., Glick, P., Expósito, F., De Lemus, S., \& Hart, J. (2007). It's for your own good: Benevolent Sexism and women's reactions to protectively justified restrictions. Personality and Social Psychology Bulletin, 33(10), 14211434. http://dx.doi.org/10.1177/0146167207304790

Orozco, A. E., Nievar, M. A., \& Middlemiss, W. (2012). Domestic violence in Mexico: Perspectives of Mexican Counselors. Journal of Comparative Family Studies, 43(5), 751-772. http://dx.doi.org/10.3138/jcfs.43.5.751

Reitzel-Jaffe, D., \& Wolfe, D. A. (2001). Predictors of relationship abuse among young men. Journal of Interpersonal Violence, 16(2), 99-115. http://dx.doi. org/10.1177/088626001016002001

Rey-Anacona, C. A. (2013). Prevalencia y tipos de maltrato en el noviazgo en adolescentes y adultos jóvenes. Terapia
Psicológica, 31(2), 143-154. http://dx.doi.org/10.4067/ S0718-48082013000200001

Rivera, L., Allen, B., Rodríguez, G., Chávez, R., \& Lazcano, E. (2007). Prevalence and correlates of adolescent dating violence: Baseline study of a cohort of 7960 male and female Mexican public school students. Preventive Medicine, 44(6), 477-484. http://dx.doi.org/10.1016/j.ypmed.2007.02.020

Russo, N. F., \& Pirlott, A. (2006). Gender-based violence concepts, methods, and findings. Annals of the New York Academy of Sciences, 1087(1), 178-205. https://doi. org/10.1196/annals.1385.024

Sakalli-Ugurlu, N., Glick, P., \& Yalcin, Z. S. (2007). Ambivalent sexism, Belief in a Just World, and empathy as predictors of Turkish students' attitudes toward rape victims. Sex Roles, 57, 889-895. http://dx.doi.org/10.1007/s11199-007-9313-2

Saunders, D. G., Lynch, A. B., Grayson, M., \& Linz, D. (1987). The Inventory of Beliefs about Wife Beating: The construction and initial validation of a measure of beliefs and attitudes. Violence and Victims, 2(1), 39-57. https://doi. org/10.1891/0886-6708.2.1.39

Shen, A. C.-T., Chiu, M. Y.-L., \& Gao, J. (2012). Predictors of dating violence among Chinese adolescents: The role of gender-role beliefs and justification of violence. Journal of Interpersonal Violence, 27(6), 1066-1089. http://dx.doi. org/10.1177/0886260511424497

Sherry, A., \& Henson, R. K. (2005) Conducting and interpreting canonical correlation analysis in personality research: A user-friendly primer. Journal of Personality Assessment, 84(1), 37-48. http://dx.doi.org/10.1207/ s15327752jpa8401_09

Sugarman, D. B., \& Frankel, S. L. (1996). Patriarchal ideology and wife-assault: A meta-analytic review. Journal of Family Violence, 11, 13-40. http://dx.doi.org/10.1007/BF02333338

Tabachnick, B. G., \& Fidell, L. S. (2012). Using multivariate statistics ( $6 .{ }^{\mathrm{a}}$ ed.). Person Education.

Tang, C. S. K., Wong, D., \& Cheung, F. M. C. (2002). Social construction of women as legitimate victims of violence in Chinese societies. Violence Against Women, 8(8), 968-996. http://dx.doi.org/10.1177/107780102400447096

Temurtas, A. (2016). Canonical correlation analysis. En C. O. Guzeller (Ed.). Multivariate statistics for all (pp.101-112). Maya Academy Publication.

Thompson, A. G. (1992). Teachers' beliefs and conceptions: A synthesis of the research. En D. A. Grouws (Ed.), Handbook of research on mathematics teaching and learning (pp. 127146). Macmillan Publishing Co, Inc.

Thompson, B. (1984). Canonical correlation analysis: Uses and interpretation. Sage.

Thompson, B. (1991). A primer on the logic and use of canonical correlation analysis. Measurement and Evaluation in Counseling and Development, 24(2), 80-95. https://psycnet. apa.org/record/1991-32137-001 
Tonsing, J. C., \& Tonsing, K. N. (2019). Understanding the role of patriarchal ideology in intimate partner violence among South Asian women in Hong Kong. International Social Work, 62(1), 161-171. http://dx.doi. org/10.1177/0020872817712566

Waltermaurer, E. (2012). Public justification of intimate partner violence: A review of the literature. Trauma, Violence, \& Abuse, 13(3), 167-175. http://dx.doi. org/10.1177/1524838012447699

Wolfe, D. A., Scott, K., Reitzel-Jaffe, D., Wekerle, C., Grasley, C., \& Pittman, A. L. (2001). Development and validation of the conflict in adolescent dating relationships inventory.
Psychological Assessment, 13(2), 277-293. http://dx.doi. org/10.1037/1040-3590.13.2.277

Zink, T., Regan, S., Jacobson, C. J., \& Pabst, S. (2003). Cohort, period, and aging effects: A qualitative study of older women's reasons for remaining in abusive relationships. Violence Against Women, 9(12), 1429-1441. http://dx.doi. org/10.1177/1077801203259231

Žukauskienė, R., Kaniušonytė, G., Bergman, L. R., Bakaitytė, A., \& Truskauskaitè-Kunevičienè, I. (2019). The role of social support in identity processes and posttraumatic growth: A study of victims of intimate partner violence. Journal of interpersonal violence. http://dx.doi. org/10.1177/0886260519836785 